\title{
Aplicabilidade dos conceitos do desenho universal e de usabilidade no desenvolvimento de produtos.
}

Applicability of the concepts of universal design and usability in product development.

FAUST, Fernanda Gomes, Mestre; Universidade Federal de Santa Catarina

fernandagfaust@gmail.com

SIERRA, Isabella de Souza; Mestre; Universidade Federal do Paraná

isa.dss@gmail.com

GOMES FERREIRA, Marcelo Gitirana; Doutor; Universidade Estadual de Santa Catarina

marcelo.gitirana@gmail.com

OKIMOTO, Maria Lúcia Leite Ribeiro; Doutora; Universidade Federal do Paraná

lucia.demec@ufpr.br

VERGARA, Lizandra Garcia Lupi; Doutora; Universidade Federal de Santa Catarina

I.vergara@ufsc.br

\section{Resumo}

Dada a importância do design universal e da usabilidade em contextos de projeto, objetivou-se realizar um mapeamento sobre os conhecimentos e práticas desses temas entre os profissionais em atividade no desenvolvimento de produto. Utilizou-se um método de análise de conteúdo a partir de entrevistas feitas com 14 graduados e atuantes em desenvolvimento de produto. Tomou-se como hipótese de que o desconhecimento destes temas acarreta a sua não utilização a qual pode ser corroborada através dos resultados obtidos. Das características dos participantes destacam-se o desconhecimento sobre o tema design universal e pouca utilização das práticas de usabilidade. A usabilidade era de domínio do conhecimento e assim utilizada, e o design universal, de menor domínio e, portanto, menos utilizado. Salientamos que a importância trazida pela integração entre os temas traria maior inclusão social e melhor desempenho de produtos, entretanto, encontrou-se na prática um contexto em que são pouco utilizados e até mesmo ignorados.

Palavras Chave: Usabilidade, design universal, desenvolvimento de produto

\section{Abstract}

Given the importance of universal design and usability in project contexts, the objective of this article was to map the knowledge and practices of these themes among professionals in product development. A content analysis method was used from interviews with 14 graduates and product development workers. It was hypothesized that the lack of knowledge of these subjects leads to their non-use which can be corroborated through the results obtained. From the characteristics of the participants stand out the lack of knowledge about universal design and little use of usability practices. Usability was of domain of knowledge and thus used, and universal design, of lesser domain and therefore, less used. We emphasize that the importance of the integration between the 
themes would bring greater social inclusion and better product performance, however, a context in which they are little used and even ignored has been found in practice.

Keywords: usability, universal design, product development

\section{Introdução}

A literatura disponibiliza, de forma geral, modelos, métodos e ferramentas de projeto para o desenvolvimento de produtos. No entanto, no desenvolvimento de produtos, a usabilidade e o design universal são assuntos geralmente delegados à sua utilização ao final do projeto (MERINO et al, 2012). Em pesquisas ainda são escassos os modelos e métodos que incluem esses temas de usabilidade e design universal de forma transversal.

O design universal juntamente com a usabilidade, tem como objetivo fornecer condições de acesso a todos os indivíduos, respeitando e considerando as suas diferenças no desenvolvimento de produtos. Os princípios do design universal podem auxiliar na obtenção de um entendimento mais completo das necessidades dos consumidores, objetivando aprimorar o produto e proporcionar uma melhor interação de todos os usuários com este produto. Estes princípios se referem ao uso equitativo, uso flexível, uso simples e intuitivo, informação de fácil percepção, tolerância ao erro, baixo esforço físico, dimensão e espaço para aproximação e uso (STORY; MUELLER; MACE, 1998).

Em pesquisa bibliográfica realizada neste estudo não foi encontrado especificamente um método que abordasse princípios do design universal durante o processo de desenvolvimento de produtos (PDP). Mas, deve-se destacar as pesquisas de Demirbilek e Demirkan (2012) que apresentam um estudo com foco em design universal que, apesar de não contemplar todo o processo de desenvolvimento do produto está focado na realização de avaliações durante o PDP utilizando no estudo a avaliação de usuários idosos nomeadamente.

Com relação à avaliação da usabilidade no PDP, destaca-se a pesquisa realizada por Araújo (2014), que apresenta uma sistemática para a avaliação da usabilidade e da experiência do usuário durante todas as fases do processo de desenvolvimento de produtos. Outros autores podem ser citados como, por exemplo: Han et al. (2001), Merino et al. (2012) e Albertazzi, Okimoto e Gomes Ferreira (2012), que realizam pesquisas na área de avaliação da usabilidade no processo desenvolvimento de produtos.

Este artigo tem como objetivo realizar um mapeamento exploratório sobre os conhecimentos e práticas dos temas design universal e usabilidade entre os profissionais em atividade no desenvolvimento de produtos. Esse mapeamento foi feito por meio de métodos de análise de conteúdo com base em um levantamento da percepção de conhecimento dos desenvolvedores de produto, coletando os dados a partir de entrevistas, quanto aos temas e uso desses temas em seu cotidiano profissional.

Acredita-se que as avaliações da usabilidade no PDP com foco no design universal auxiliam no desenvolvimento de produtos para maior inclusão social, visto que maiores números de usuários utilizarão os produtos com mais facilidade e autonomia em sua vida diária, resultando em maior qualidade de vida. Apesar disso, parece que são pouco utilizados e, às vezes, ignorados completamente no processo. Por este motivo tomou-se como hipótese que o desconhecimento dos 
temas acarreta a não utilização dos mesmos pelos profissionais.

\section{Fundamentação Teórica}

O Processo de Desenvolvimento de Produtos (PDP), para Rozenfeld et al (2006), é um conjunto de atividades com as quais se busca chegar às especificações de projeto de um produto e de seu processo de produção, de forma que a manufatura seja capaz de produzi-lo. Esse processo também envolve as atividades de acompanhamento do produto após o lançamento para serem realizadas as mudanças necessárias nessas especificações.

Um tema que aborda conceitos para a melhoria do acesso das pessoas aos produtos e que deve ser trabalhado juntamente ao PDP é o Design Universal. Pois uma vez que visa-se desenvolver produtos e serviços que possam ser utilizados pelo maior número de pessoas, o design universal, termo inicialmente usado nos Estados Unidos pelo arquiteto Ron Mace em 1985. Tendo sido definido pelos autores como o projeto de produtos e ambientes que possam ser usados pela maior extensão possível de pessoas de todas as idades e habilidades, respeitando a diversidade humana e promovendo a inclusão de todas as pessoas em todas as atividades de sua vida. Dessa forma, pode-se acomodar uma ampla faixa de usuários, incluindo crianças, idosos, pessoas com deficiência e pessoas com tamanho ou proporções diversas (STORY; MUELLER; MACE, 1998).

As abordagens convencionais de desenvolvimento de produto, na lógica da produção em massa, têm feito com que algumas pessoas dependam de dispositivos de apoio para realizar suas atividades cotidianas e excluem outras, por exemplo, aquelas com limitações físicas ou cognitivas (DONG, 2007). Os princípios do design universal auxiliam no desenvolvimento de produtos considerando as diferentes habilidades e capacidades dos usuários.

O design universal apresenta quatro princípios: 1) acomodar uma grande gama antropométrica, e isto significa acomodar pessoas de diferentes dimensões: altas, baixas, em pé, sentadas e em diferentes necessidades de posição para o uso do produto, etc.; 2) reduzir a quantidade de energia necessária para utilizar os produtos e o meio ambiente; 3 ) tornar o ambiente e os produtos mais abrangentes e, 4) a ideia do desenho de sistemas, no sentido de pensar em produtos e ambientes como sistemas, que talvez tenham peças intercambiáveis ou a possibilidade de acrescentar características para as pessoas que têm necessidades especiais (STEINFELD, 1994).

Além da adaptação de produtos, Teixeira, Okimoto e Heemann (2015) abordam a aplicação do design universal em linhas de produção, alternativa para facilitar a inclusão e o aproveitamento das capacidades das pessoas com deficiências e Guazzelli et al (2013) defende que o design universal busca formas de garantir a inclusão social das pessoas com deficiência ou com mobilidade reduzida.

Outro tema a ser considerado e que afeta no PDP, colaborando na determinação do sucesso dos produtos, é a usabilidade. Para Preece, Rogers e Sharp (2005) muitos produtos interativos não são necessariamente projetados tendo o usuário em mente e sim para realizar funções. A usabilidade pode auxiliar na obtenção de um entendimento mais completo das necessidades dos consumidores, as avaliações de usabilidade auxiliam na ponderação do uso e da relação do produto com o usuário, objetivando aprimorar o produto e proporcionar uma melhor interação do usuário com o mesmo. Uma vez que como afirmam Gonzalez-Sanchez e Gil-Iranzo (2013) uma boa experiência pode se tornar um fator decisivo para o sucesso de um produto em um mercado cada vez mais saturado e competitivo.

A usabilidade é considerada como a habilidade do usuário em realizar a tarefa com sucesso 
(JORDAN, 1998). A estreita relação entre as funções dos produtos e a usabilidade consolida a importância deste fator em aspectos simbólicos e emocionais do produto. Sendo importante compreender em que ambientes técnicos, físicos e sociais o produto será utilizado, e como isso pode influenciar seu uso (ABNT, 2002; JORDAN, 1998; NIELSEN, 1993).

Os autores Djajadiningrat, Matthews e Stienstra (2007) apresentam um estudo que trata da influência da estética na usabilidade de produtos e salientam que assim como a "má usabilidade" pode influenciar negativamente a percepção estética do produto, o inverso também pode ocorrer durante a interação do usuário com o produto. E, de acordo com Khalid e Helander (2006), as emoções positivas podem melhorar sistematicamente o processo cognitivo do usuário, gerando satisfação. Correia e Soares (2007) apresentam dados de aspectos relacionados à segurança e qualidade dos produtos que devem ser tratados com maior seriedade pelos órgãos e agentes responsáveis, devido ao alto índice de falhas por parte dos produtos desenvolvidos e fabricados nos dias atuais no que tange à usabilidade, à qualidade e à segurança.

Percebe-se que as avaliações de usabilidade e desenvolvimento de produto com foco no Design Universal podem auxiliar na obtenção de um entendimento mais completo das necessidades dos indivíduos, proporcionando uma melhor experiência de uso e interação dos indivíduos, com uma consequente melhoria da sua autonomia, da qualidade de vida e do aumento do sucesso dos produtos para as empresas.

\section{Método}

A pesquisa teve como base um estudo exploratório qualitativo (RICHARDSON, 2012), em forma de entrevistas e análise de conteúdo (GIL, 2002). Foram efetuadas entrevistas no segundo semestre de 2017 com 14 indivíduos graduados e com profissões relacionadas ao desenvolvimento de produto, no estado de Santa Catarina. Nas entrevistas buscou-se levantar dados sobre a percepção desses indivíduos sobre os temas design universal e usabilidade, metodologias de projeto, a identificação do seu conhecimento sobre os temas e o uso em seu cotidiano profissional.

A coleta de dados foi realizada na Universidade Federal de Santa Catarina (UFSC), em uma sala previamente reservada. Os participantes foram selecionados por conveniência desde que aceitassem participar voluntariamente da pesquisa, assinando um Termo de Consentimento de Livre Esclarecido (TCLE) que incluía a permissão de gravação de voz, e se enquadrassem aos requisitos de formação, estar graduado, e trabalhar na área de desenvolvimento de produtos. As entrevistas foram agendadas previamente com os voluntários em horário que melhor atendesse sua disponibilidade, sendo previsto o tempo de duração de aproximadamente 10 minutos. As entrevistas foram gravadas (voz) para posterior transcrição e análises qualitativas. Utilizou-se o método de análise de conteúdo nos dados coletados pelas entrevistas.

A técnica de análise de conteúdo enquadra-se na descrita por Bardin (2011) A qual utiliza-se de procedimentos sistemáticos e objetivos de descrição do conteúdo das mensagens com o uso de indicadores, que podem ser quantitativos ou qualitativos, mas que permitam a inferência de conhecimentos relativos às condições de produção/recepção das variáveis inferidas. Esta técnica é dividida em três etapas: 1) a pré-análise; 2) a exploração do material e; 3) o tratamento dos resultados, que foi constituída pela inferência e a interpretação (BARDIN, 2011).

Na pré-análise foi verificado o objetivo da pesquisa "realizar um mapeamento sobre os conhecimentos e práticas dos temas, design universal e usabilidade, entre os profissionais em 
atividade no desenvolvimento de produto", para desenvolver as perguntas que foram feitas nas entrevistas e estabelecidos critérios para seleção dos entrevistados (BARDIN, 2011). As questões dividiram-se em duas partes. A primeira parte que foram questões introdutórias demandaram quanto à idade, sexo, formação acadêmica, tempo de formação acadêmica e cidade de residência.

Após estas informações gerais sobre os entrevistados, iniciou-se a segunda parte que se refere a entrevista com as questões relativas aos temas (Quadro 1). As entrevistas foram gravadas em áudio e em seguida transcritas de forma fidedigna para posterior análise dos dados.

Quadro 1 - Questões da entrevista

\begin{tabular}{ll} 
No & Questões \\
\hline 1 & Qual seu entendimento de Design Universal? \\
\hline 2 & $\begin{array}{l}\text { Considerando que um dos princípios básicos do design universal é o desenvolvimento de projetos para todos } \\
\text { ou o maior número de pessoas. Você já realizou disciplinas ou projetos utilizando os conceitos de Design } \\
\text { Universal?* }\end{array}$ \\
\hline 3 & E porque você optou por utilizar ou não os princípios? \\
\hline 4 & Qual o seu entendimento de Usabilidade? \\
\hline 5 & $\begin{array}{l}\text { Considerando que as avaliações da usabilidade auxiliam na interação dos produtos com os usuários. Você já } \\
\text { realizou disciplinas ou projetos utilizando os conceitos de Usabilidade?* }\end{array}$ \\
\hline 7 & $\begin{array}{l}\text { E porque você optou por utilizar ou não os princípios? } \\
\text { desenvolvênimento de produtos, de que forma? Se não, o porquê você acredita que não possam auxiliar? }\end{array}$ \\
\hline *Realizar disciplinas nesse contexto significou cursar disciplinas.
\end{tabular}

Fonte: Elaborado pelos Autores (2018)

Depois de realizada a transcrição literal e fidedigna das entrevistas a análise foi dividida em duas partes: 1) Foram classificadas as questões de número 1 e de número 4 que referem-se sobre o entendimento do entrevistado sobre os assuntos questionados em: "entendimento correto do tema", para quando o entrevistado responde corretamente, "entendimento parcial do tema", quando o entrevistado responde sobre o tema mesmo que não totalmente correto e, "entendimento incorreto do tema", quando exposto o não conhecimento ou a resposta não condizente com a realidade. 2) A segunda parte foi a exploração do conteúdo segundo Guerra (2010). Conforme recomenda o autor explorando o conteúdo de forma a encontrar temáticas e categorias para serem posteriormente analisadas. As categorias escolhidas foram as temáticas do presente trabalho: "Design universal e Usabilidade".

Definidas as temáticas que nortearam a avaliação, iniciou-se o processo de análise dos dados coletados. Na presente pesquisa, utilizou-se do Microsoft Excel e de uma ferramenta contadora de palavras repetidas, wordclouds.com, como apoio para o tratamento e análise dos dados que estão apresentados na seção dos resultados.

\section{Resultados e Discussão}

Os resultados expostos na presente pesquisa refletem o contexto de um grupo específico, observado neste estudo. Para a obtenção de dados representativos e faz-se necessário um estudo mais amplo, com uma amostra com maior número de participantes. Apresenta-se a seguir o perfil dos entrevistados e o entendimento dos conceitos "design universal", "usabilidade" e da relação de ambos, tanto na formação acadêmica quanto na atuação profissional aplicados ao PDP. 


\subsection{Estratificação dos dados coletados}

Foram entrevistados 14 sujeitos sendo sete mulheres e sete homens com faixa etária de 23 a 40 anos. Destes, $14 \%$ eram designers gráficos, $72 \%$ designers de produto ou industriais, $7 \%$ tinham formação de design sem habilitação específica e 7\%, administradores que desenvolvem a prática de desenvolvimento de produto, totalizando $93 \%$ dos entrevistados tinham formações teóricas de desenvolvimento de produto (Gráfico 1). Sua média de tempo de prática profissional é de 3,14 anos, sendo o menor tempo de prática um ano e o maior tempo oito anos.

Na pesquisa houveram três outros entrevistados com formação em engenharia mecânica e engenharia elétrica que desistiram de responder a entrevista (e não foram contabilizados), pois, por mais que trabalhassem com desenvolvimento de produto na prática, não se sentiram aptos em responder os temas e assim não participaram na pesquisa.

Gráfico 1 - Amostra por profissão dos entrevistados

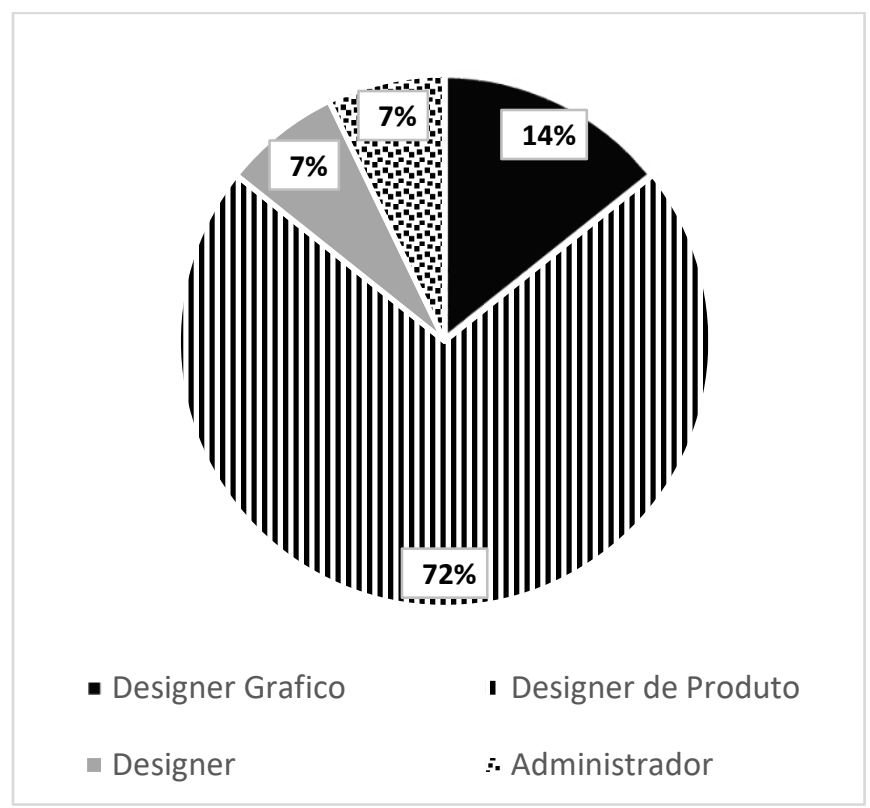

Fonte: Elaborado pelos Autores (2018)

\subsection{Entendimento do Conceito de Design Universal}

O conceito aqui utilizado para o termo é o seguinte: O Design Universal deve acomodar a todos, ou o maior número de pessoas possíveis sem se tratar de uma tecnologia específica para uma necessidade específica (STORY; MUELLER; MACE, 1998; STEINFELD, 1994). Dos entrevistados, 79\% mostraram ter um conceito geral do tema correto, 14\% apresentaram parcialmente conhecer o tema e apenas um entrevistado respondeu não ter conhecimento do tema.

Em relação ao conhecimento dos conceitos da temática, as respostas foram divididas em três subcategorias, como apresenta o Quadro 2. A primeira categoria referente ao conceito de uso equitativo "todos", foi utilizado por 5 participantes. O segundo conceito é a temática de acesso e adaptação de espaço para acesso também comentada por 5 participantes. $E$ a última temática é a minimização de barreiras que somente foi citada por dois participantes. 
Quadro 2 - Expressões sobre definição de design universal

\begin{tabular}{lll} 
Categoria & Palavras e Expressões & Participantes \\
\hline Todos & "para qualquer um"; "para todos"; “o maior número de pessoas" & E1; E2; E8; E9; E13 \\
\hline Acesso/Adaptação & "dar acesso"; "adaptar a todos"; “ser acessível; “dar acessibilidade” & E1; E2; E7; E12; E14 \\
\hline Minimizar Barreiras & "minimizar barreiras"; "mínimo de barreiras" & E1; E10 \\
\hline
\end{tabular}

Fonte: Elaborado pelos Autores (2018)

Quanto às expressões identificadas em relação ao design universal, no Quadro 2, pode-se perceber que a maioria dos entrevistados expressou o conhecimento do tema de projeto de design universal como um projeto para a totalidade de pessoas; poucos colocaram como uma maneira de inclusão, mas apenas quando se tem a dificuldade de acesso limitada por questões físicas ou cognitivas. Nessa mesma análise, dos termos que apareceram em maior número e que compõe o conceito, foram "para todos" e "pessoas". Conclui-se que o conceito geral de que design universal é para todas as pessoas, é de fato assimilado, não sendo apenas entendido por alguma deficiência ou acessibilidade necessária. Como o estudo não aprofundou de forma muito detalhada com questões sobre o conhecimento adquirido a respeito do design universal e de usabilidade, não foi possível avaliar o potencial de conhecimento adquirido. Além disso os sujeitos se limitaram a fornecer respostas curtas e superficiais.

Para uma análise ampla dos termos utilizados, a Figura 1 expõe o painel de palavras e expressões dos participantes. As palavras maiores se referem às que tiveram mais citações: "para todos", "maior número de pessoas" e "acessibilidade" foram as mais faladas.

Figura 1 - Síntese de conceito de design universal obtida no estudo exploratório

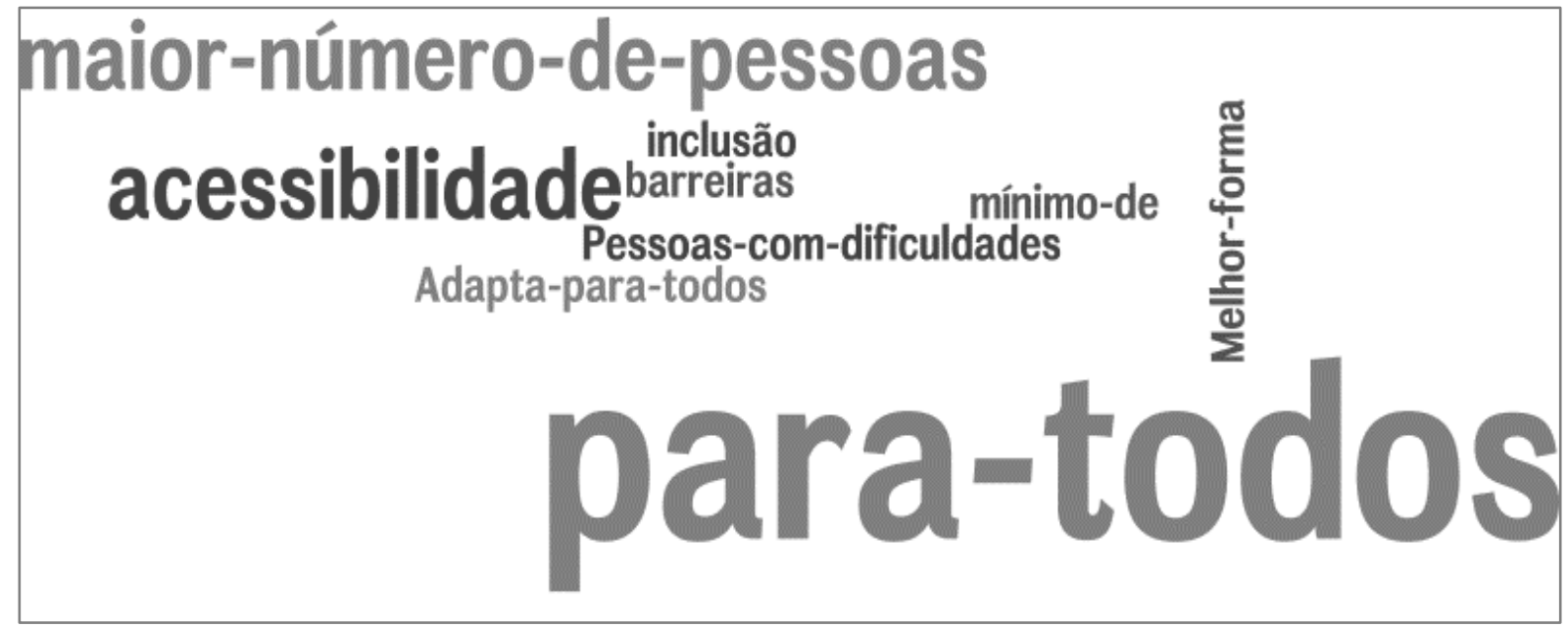

Fonte: Elaborado pelos Autores (2018)

\subsubsection{Conhecimento e Aplicação do Design Universal em sua Formação e Profissional}

Quando questionados se já tinham conhecimento prévio do tema design universal, por meio de teoria ou prática profissional, $50 \%$ dos entrevistados disseram ter tido por meio disciplinas cursadas ou no desenvolvimento de projetos dentro da academia. Na atividade profissional, 36\% disseram utilizar os conceitos, mesmo que não abordados na formação acadêmica, e, outros $64 \%$, 
mencionaram não utilizar o conceito do design universal em atividades de projeto de produto. Ao serem questionados sobre a opção de utilizar, ou não, os princípios do design universal, 64\% não optaram em utilizar os princípios em atividades de projeto justificando conforme o Quadro 3:

Quadro 3 - Justificativa dada pelos entrevistados que não optaram por utilizar design universal

\section{Entrevistado Não Optaram por Design Universal}

\begin{tabular}{ll}
\hline E1 & "Eu não tive muita visão nisso." \\
\hline E3 & "Pois a etapa de projeto que envolve essa parte, eu não fazia parte da equipe." \\
\hline E8 & "Por não conhecimento." \\
\hline E13 & "Por não estar no escopo do projeto." \\
\hline
\end{tabular}

Fonte: Elaborado pelos Autores (2018)

Dos dados obtidos, nove entrevistados disseram não utilizar o Design Universal em suas atividades profissionais. Destes, sete possuem conhecimento correto do tema, um tem conhecimento parcial e outro não tem conhecimento. Os outros $36 \%$ dos entrevistados que optaram por utilizar os conceitos do design universal na sua atividade profissional, quatro deles responderam corretamente os termos e um deles parcialmente. A falta de conhecimento e a sensação de falta de oportunidade de aplicação dos temas nos projetos trabalhados ou pelo cargo que ocupavam são as justificativas mais comuns. Percebe-se que, de um modo geral, mesmo tendo conhecimento sobre o design universal, a vida profissional é um fator decisivo na aplicabilidade do desenvolvimento de projetos com os princípios de design universal.

\subsection{Entendimento do Conceito de Usabilidade}

O conceito de usabilidade utilizado é o proposto por Jordan (1998) e a ISO-NBR 924111 (2002) que é a propriedade de interação entre o produto, o usuário e a tarefa, ou um conjunto de tarefas e o sucesso na sua realização. Todos os entrevistados souberam responder corretamente o conceito de usabilidade, demonstrando que o tema é comum e conhecido, até mesmo para quem não tinha uma formação específica na área de desenvolvimento de produto.

Sobre o conhecimento específico de usabilidade, as respostas foram subdivididas em categorias de conceito, sendo estas: "facilidade ou adequação do uso", "ergonomia" e "interação", como apresenta o Quadro 4.

Quadro 4- Dados coletados sobre conceitos de usabilidade.

\begin{tabular}{|c|c|c|}
\hline Categoria & Expressões & Entrevistados \\
\hline $\begin{array}{l}\text { Facilidade } \\
\text { adequação }\end{array}$ & $\begin{array}{l}\text { "Precisava estar adequado"; "Fácil uso"; "projeto fácil”; "encaixa perfeito entre } \\
\text { usuário e produto"; “fácil uso"; "o quão fácil é o uso"; "intuitiva". }\end{array}$ & 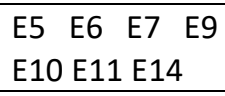 \\
\hline Ergonomia & "Ergonomia"; "é parte da ergonomia"; "trazer mais conforto ao usuário". & E1 E2 E4 E11 \\
\hline Interação & $\begin{array}{l}\text { "interação do usuário"; "maneira de uso"; "permitir uma melhor interação"; } \\
\text { "interface física ou cognitiva"; "estudo do uso, interação fácil”; "uso"; "manuseio"; } \\
\text { "que o usuário consiga interagir". }\end{array}$ & 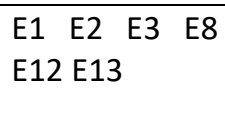 \\
\hline
\end{tabular}

Fonte: Elaborado pelos Autores (2018)

A Figura 2 apresenta o painel de palavras relativo ao entendimento dos participantes do termo usabilidade. As palavras mais citadas foram: "Interação", "bom-uso", "fácil uso". 
Figura 2 - Síntese de conceito de usabilidade obtida no estudo exploratório

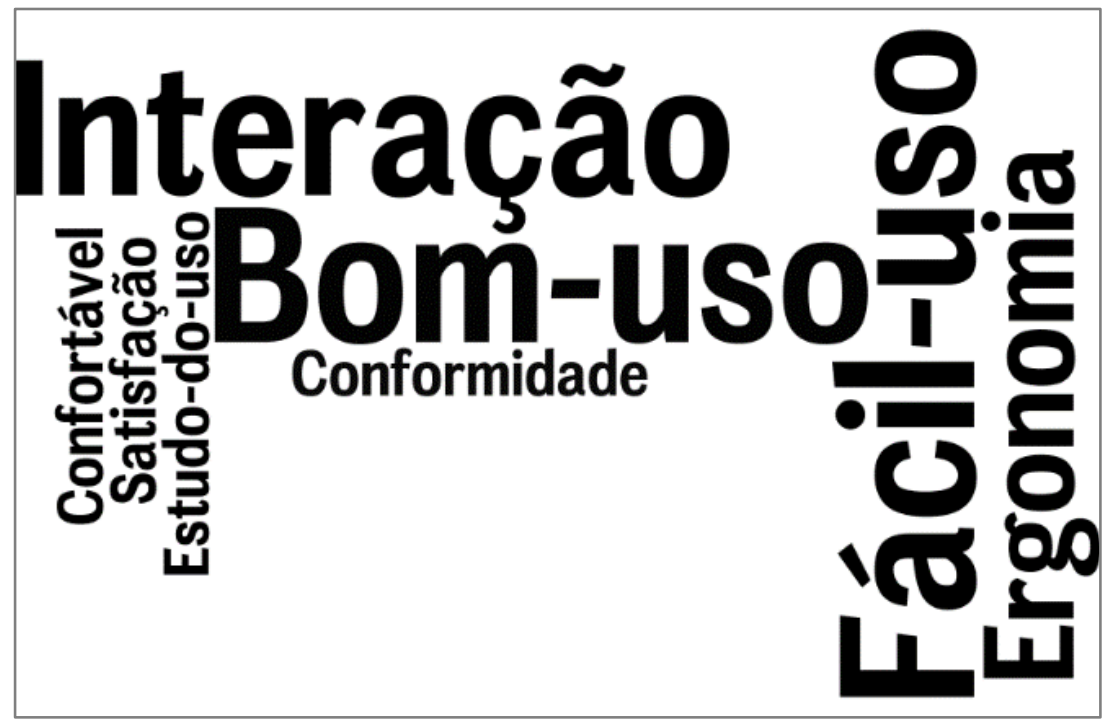

Fonte: Elaborado pelos Autores (2018)

Uma das interpretações que pode ser dada ao conhecimento integral sobre o assunto é o fato da amostra ser majoritariamente composta de designers. Todos os sujeitos com formação acadêmica em design tiveram a disciplina de ergonomia na graduação onde a usabilidade é tratada. Esse também pode ser o motivo pelo qual o design universal não era conhecido por todos, sendo este tema não incluído na maioria das grades curriculares dos cursos de design dos entrevistados.

\subsubsection{Conhecimento e Aplicação da Usabilidade em sua Formação e Profissional}

Sobre os questionamentos do conhecimento e a aplicação da usabilidade em disciplinas e projetos, as respostas foram $100 \%$ afirmativas. Porém, não foi possível distinguir na análise de conteúdo desta pergunta se foi na formação acadêmica ou na atividade profissional, para que pudesse ser dada uma resposta quantitativa. Apenas um entrevistado que não tinha formação em design respondeu ter utilizado como "conformidade de requisitos".

Quando questionados porque optaram ou não por utilizar os princípios ou ferramentas da usabilidade, conforme aponta o Quadro 5, as justificativas foram apontadas como um fator inerente ao projeto e a profissão.

Quadro 5 - Justificativa de entrevistados que optaram por utilizar usabilidade

\begin{tabular}{ll} 
Entrevistado & Optaram por Usabilidade \\
\hline E01 & "Sinceramente para melhorar a experiência que alguém vai ter com o produto que eu projetei e \\
& assim melhorar o meu projeto." \\
\hline E07 & a pessoa evitar que a pessoa fique irritada ou não consiga encontrar uma informação. Para evitar que \\
& "Ah, porque usabilidade! Se você fizer um projeto sem usabilidade você basicamente não consegue \\
E09 & atingir o seu cliente e ai você estraga tudo que você tinha feito a partir do projeto." \\
& "Justamente pra que o trabalhador consiga desempenhar a tarefa dele da maneira menos \\
trabalhosa! Com menos fadiga, com eficiência e eficácia... eu trabalhei dentro da visão ergonômica."
\end{tabular}

Fonte: Elaborado pelos Autores (2018) 
Ao analisar as respostas percebe-se que os entrevistados possuem um conceito definido de usabilidade e de sua aplicabilidade. A entrevista procurou não abordar quais os métodos ou técnicas de usabilidade que os desenvolvedores utilizavam, porém foi possível perceber que os entrevistados dão importância ao conceito na atividade profissional, e que de fato a usabilidade apresenta resultados positivos.

\subsection{Compreensão da união dos temas designs universal e usabilidade}

Por fim, a última pergunta da entrevista refere-se sobre como a união e interação dos temas design universal e usabilidade poderia ser utilizada no desenvolvimento de produtos. Todos os entrevistados responderam afirmando que acreditavam nesta aplicação de integração dos dois temas. Quando questionados de que forma, os indivíduos expuseram suas opiniões com pouca maturidade sobre a realidade científica e com conceitos não muito lógicos. No Quadro 6 são apresentadas as posições dos entrevistados sobre a união do tema.

Mesmo que acreditem que seja possível, alguns levantaram alguns pontos como o aumento de tempo de projeto, outros citaram a melhoria das interações físicas dos produtos e finalmente que seria até um pouco estranho estar utilizando os dois conceitos de forma separada.

Quadro 6 - Trechos de entrevistados que optaram por utilizar usabilidade em conjunto com design universal

\begin{tabular}{ll} 
Entrevistado & União dos temas \\
\hline E02 & "...fazendo projeto mais abrangente possível para todos seguindo esses conceitos de interação com \\
& o usuário, tanto física quanto mentalmente. " \\
\hline E03 & "...teórica chegaria em o mais próximo de um produto ideal para uma população diversificada quanto \\
& é o mundo. Você vai atender diversas faixas etárias e limitações físicas." \\
\hline E07 & $\begin{array}{l}\text { "...no meu entendimento pelo que eu e lembro dos princípios e eu acho que um acaba } \\
\text { complementando o outro, eu acho que é meio estranho estar separando usabilidade de design } \\
\text { universal. " }\end{array}$ \\
\hline E10 & $\begin{array}{l}\text { “...de forma a minimizar... eu acho que ela aumenta o tempo de projeto, só que elas minimizam a } \\
\text { chance de frustração e erro do produto. " }\end{array}$ \\
\hline
\end{tabular}

Fonte: Elaborado pelos Autores (2018)

Numa análise do Quadro 6, entende-se que os entrevistados têm conhecimento superficial dos temas, sendo usabilidade mais conhecida do que o design universal e sua prática de uso também maior. Os entrevistados demonstram importar-se com a utilização dos temas juntos e demonstram a dificuldade de colocar em prática a utilização dos conceitos. Percebe-se esta dificuldade especialmente quando tratam do tema como complementariedade, revelando que acreditam que o uso dos conceitos poderá aumentar o tempo de projeto, dificultando sua prática.

\section{Conclusão}

Conclui-se através deste estudo exploratório que as técnicas utilizadas de análise de conteúdo permitem revelar alguns dados importantes quanto a falta de conhecimento sobre design universal e usabilidade e seu potencial para as estratégias do desenvolvimento de produto.

Esta técnica de entrevista estruturada focada nos temas e técnicas de análise de conteúdo possibilitaram encontrar também algumas características relativas aos conhecimentos dos participantes. São elas: o desconhecimento ou pouco conhecimento do tema design universal, especialmente para usos pragmáticos e específicos, sendo o termo compreendido, mas não sua 
aplicação; o conhecimento e aplicação de avaliações de usabilidade em projetos com foco especifico ao cumprimento de normas e adequação dos produtos; e a percepção e compreensão das possibilidades geradas pela junção dos temas, mas considerados utópicos.

Ao remeter-se à hipótese levantada neste estudo de que o desconhecimento dos temas acarreta a não utilização dos mesmos pelos profissionais, encontraram-se fortes corroborações. 0 conceito de usabilidade é conhecido e deste modo utilizado, e o design universal também por ser desconhecido é consequentemente menos utilizado.

Dentro da amostra do estudo, identificaram-se lacunas no conhecimento do design universal e dificuldade em sua aplicabilidade. Um dos fatores que se acredita ter influenciado está relacionado à ausência de requisitos de projeto voltados para o tema.

Quanto à usabilidade, todos os entrevistados mostraram ter conhecimento sobre o que seria usabilidade associada ao uso, demonstrando que não é um tema desconhecido. Entretanto, ao se questionar sobre a sua utilização, eles demonstram não utilizar métodos ou ferramentas para a sua aplicabilidade, mesmo quando alguns trataram do tema com grande importância na prática profissional.

A importância trazida a luz nesse estudo, na qual a integração entre os temas traria maior inclusão social e consequentemente maior qualidade de vida e maior chance de sucesso para os novos produtos. No entanto, encontrou-se um contexto em que são pouco utilizados e, às vezes, ignorados completamente no processo projetual.

Para trabalhos futuros acredita-se que seria interessante apresentar um questionário antes das entrevistas sobre disciplinas que já realizaram. Então, a partir daí, teríamos informações da base teórica dos entrevistados, que demonstraram mais conhecimento sobre usabilidade por já ter tido contato com ela na universidade ou no contexto onde houve o aprendizado.

Outra questão a ser abordada futuramente é conseguir uma amostra maior e com maior abrangência de cursos de graduação como engenheiros, arquitetos e outros projetistas, e então comparar seu conhecimento e entendimento sobre os temas de desenvolvimento de projeto.

\section{Referências}

ABNT.ISO-NBR 9241-11: requisitos ergonômicos para trabalho de escritórios com computadores: parte 11 - orientações sobre usabilidade. Rio de Janeiro, 2002.

ALBERTAZZI, Deise; OKIMOTO, Maria Lucia; GOMESFERREIRA, Marcelo Gitirana. Developing an usability test to evaluate the use of augmented reality to improve the first interaction with a product. Work, [s.I.], v. 41, n. 1, p.1160-1163, 2012. IOS Press. http://dx.doi.org/10.3233/WOR2012-0297-1160.

ARAÚJO, F. S. Avaliação da experiência do usuário: uma proposta de sistematização o processo de desenvolvimento de produtos. Tese de doutorado. Programa de Pós- Graduação em Engenharia de Produção da Universidade Federal de Santa Catarina, 2014.

BARDIN, L. Análise do Conteúdo. São Paulo: Edições 70, 2011.

CORREIA, Walter Franklin Marques; SOARES, Marcelo Márcio. Segurança do Produto: Uma Investigação na Usabilidade de Produtos de Consumo. Estudos em Design, Rio de Janeiro, v. 15, n. 2, p.1-20, 2007. 
DEMIRBILEK, Oya; DEMIRKAN, Halime. Universal product design involving elderly users: a participatory design model. Applied Ergonomics, [s.I.], v. 35, n. 4, p.361-370, jul. 2004. Elsevier BV. http://dx.doi.org/10.1016/j.apergo.2004.03.003.

DJAJADININGRAT, Tom; MATTHEWS, Ben; STIENSTRA, Marcelle. Easy doesn't do it: skill and expression in tangible aesthetics. Personal And Ubiquitous Computing, [s.l.], v. 11, n. 8, p.657-676, 17 mar. 2007. Springer Nature. http://dx.doi.org/10.1007/s00779-006-0137-9.

DONG, Hua. Shifting Paradigms in Universal Design. Lecture Notes In Computer Science, [s.I.], p.6674, 2007. Springer Berlin Heidelberg. http://dx.doi.org/10.1007/978-3-540-73279-2_8.

GIL, A.C. Como elaborar projetos de pesquisa. 4ạed. São Paulo: Editora Atlas, 2002.

GONZALEZ-SANCHEZ, J.-L.; GIL-IRANZO, R.-M. ** Hedonic and multicultural factors in product design that improve the user experience. Profesional de la Informacion, v. 22, n. 1, p. 26- 35, Jan-Feb 2013.

GUAZZELLI, Anna Marina Alonso y Alonso et al. Inclusão e Acessibilidade em edifícios de uso coletivo:: O desenho universal e o sistema de ensino de Presidente Prudente. Estudos em Design, Rio de Janeiro, v. 21, n. 1, p.1-16, 2013.

GUERRA, I. C. Pesquisa qualitativa e análise de conteúdo: sentidos e formas de uso. Cascais: Principia, 2010.

HAN, Sung $\mathrm{H}$. et al. Evaluation of product usability: development and validation of usability dimensions and design elements based on empirical models. International Journal of Industrial Ergonomics, [s.I.], v. 26, n. 4, p.477-488, out. 2000. Elsevier BV. http://dx.doi.org/10.1016/s01698141(00)00019-6.

JORDAN, P. W. An introduction to usability. London: Taylor \& Francis, 1998.

KHALID, H. M.; HELANDER, M. G.. Customer Emotional Needs in Product Design. Concurrent Engineering, [s.l.], v. 14, n. 3, p.197-206, set. 2006. SAGE Publications. http://dx.doi.org/10.1177/1063293x06068387.

MERINO, Giselle Schmidt Alves Díaz et al. Usability in Product Design - The importance and need for systematic assessment models in product development - Usa-Design Model (U-D) @. Work, [s.I.], v. 41, n. 1, p.1045-1052, 2012. IOS Press. http://dx.doi.org/10.3233/WOR-2012-1011-1045.

NIELSEN, J. Usability engineering. Boston, USA: Academic, 1993.

PREECE, J.; ROGERS, Y.; SHARP, H. Design de interação: além da interação homem-computador. Porto Alegre: Bookman, 2005.

RICHARDSON, R. J. Pesquisa Social: métodos e técnicas. 3ạed. São Paulo: Atlas, 2012.

ROZENFELD, H. et al. Gestão de desenvolvimento de produtos: uma referência para a melhoria do processo. 2. ed. São Paulo: Saraiva, 2006.

STEINFELD, E. Arquitetura através do desenho universal. In: curso básico sobre acessibilidade ao meio físico e vi seminário sobre acessibilidade ao meio físico. Brasília: Corde, 1994.

STORY, Molly Follette; MUELLER, James; MACE, Ronald. The Universal Design File: Designing for People of All Ages and Abilities. 2. ed. Raleigh North Carolina: The Center For Universal Design, 1998

TEIXEIRA, Edson Sidnei Maciel; OKIMOTO, Maria Lucia Leite Ribeiro; HEEMANN, Adriano. Design 
Universal para Inclusão de Pessoas com Deficiência em Linhas de Produção Industriais: análise estruturada de publicações. Estudos em Design, Rio de Janeiro, v. 23, n. 2, p.133-149, 2015.

\section{Agradecimentos}

Às instituições: UFSC e UDESC; e as de fomento à pesquisa: Capes e CNPq. 